\title{
Double-Scope Peroral Endoscopic Myotomy (POEM) for Esophageal Achalasia: The First Trial of a New Double-Scope POEM
}

\author{
Hee Jin Hong', Ga Won Song ${ }^{1,2}$, Weon Jin Ko ${ }^{1,2}$, Won Hee Kim ${ }^{1,2}$, Ki Baik Hahm ${ }^{1,2}$, Sung Pyo Hong ${ }^{1,2}$ and Joo Young Cho ${ }^{1,2}$ \\ ${ }^{1}$ Department of Internal Medicine, ${ }^{2}$ Digestive Disease Center, CHA Bundang Medical Center, CHA University, Seongnam, Korea
}

With the accumulation of clinical trials demonstrating its efficacy and safety, peroral endoscopic myotomy (POEM) has emerged as a less invasive treatment option for esophageal achalasia compared with laparoscopic Heller myotomy. However, the difficulty in determining the exact extent of myotomy, a critical factor associated with the success and safety of the procedure, remains a limitation. Although the various endoscopic landmarks and ancillary techniques have been applied, none of these has been proven sufficient. As a solution for this limitation, the double-scope POEM technique with a second endoscope to assure the exact length of the submucosal tunnel has been applied since 2014. Before double-scope POEM was introduced, the second endoscope was applied only to confirm the accuracy of the procedure. In the present study, we performed double-scope POEM in the treatment of esophageal achalasia through a novel procedure of simultaneous application of the second endoscope to assist in the conventional POEM procedure.

Clin Endosc 2016;49:383-386

Key Words: Peroral endoscopic myotomy; Esophageal achalasia; Double-scope peroral endoscopic myotomy; Novel approach

\section{INTRODUCTION}

Esophageal achalasia is a motility disorder featured with esophageal aperistalsis and impaired relaxation of the lower esophageal sphincter (LES) during swallowing. ${ }^{1}$ Before the first introduction of peroral endoscopic myotomy (POEM) achalasia by Inoue et al. ${ }^{2}$ (Showa University Northern Hospital, Japan), most cases of achalasia was treated with either repeated endoscopic dilatation or injection therapy of botulium toxin, and surgical myotomy was performed in case of insufficient medical treatment. ${ }^{2,3}$ Currently, POEM therapy is preferred owing to its safety and effectiveness, as indicated by the increasing number of reports on POEM treatment worldwide.

Received: August 29, 2015 Accepted: September 21, 2015

Correspondence: Joo Young Cho

Digestive Disease Center, CHA Bundang Medical Center, CHA University, 59 Yatap-ro, Bundang-gu, Seongnam 13496, Korea

Tel: +82-31-780-5005, Fax: +82-31-780-5000, E-mail: cjy6695@dreamwiz.com

(cc) This is an Open Access article distributed under the terms of the Creative Commons Attribution Non-Commercial License (http://creativecommons.org/ licenses/by-nc/3.0) which permits unrestricted non-commercial use, distribution, and reproduction in any medium, provided the original work is properly cited.
Hence, an important determining factor of the safety and efficacy of POEM is the identification of the extent of the submucosal tunnel and myotomy. Determining the exact extent of myotomy in the submucosal tunnel is essential. If the length of the myotomy is too short, the procedure is ineffective, whereas if the length of the myotomy is too long, there is a higher risk of complications such as perforation or bleeding. ${ }^{4}$ Currently, several endoscopic landmarks such as palisading vessels at the esophagogastric (EG) junction and the circular bundle of LES fibers ${ }^{5}$ have been used as landmarks for the POEM procedure. These landmarks are difficult to use and inaccurate. Their critical limitation is the difficulty of determining the exact extent of the submucosal tunnel.

As a solution for the limitations of POEM, double-scope POEM was invented and applied by a research group. Double-scope POEM enables appropriate identification of the cardia in the retroflexed position by using a second ultrathin gastroscope. A recent randomized controlled trial that demonstrated its safety, accuracy, and simplicity, and proved that it allows for complete gastric myotomy was published in Japan. ${ }^{6}$ However, in the trial, the second endoscope was only used to confirm the extent of myotomy after completion of 
the myotomy, not during the POEM procedure.

Recently, we applied double-scope POEM in patients with achalasia in a simultaneous intervention using two endoscopes for POEM. Through this trial, we nominated double-scope POEM as an alternative to the previous double-scope POEM based on a similar principle but a quite different procedure.

\section{CASE REPORT}

A 19-year-old woman without any significant medical history presented with a 1-year history of dysphagia. On the initial esophagogastroduodenoscopy on admission, a dilated esophagus and retained fluid and food materials were observed. In addition, penetration of the scope was difficult, as resistance was experienced when passing the gastroscope through the
EG junction (Fig. 1A). High-resolution manometry showed an elevated LES pressure $(63.7 \mathrm{~mm} \mathrm{Hg}$ ) and incomplete relaxation $(19.9 \mathrm{~mm} \mathrm{Hg})$. The integrated relaxation pressure was also elevated at $33 \mathrm{~mm} \mathrm{Hg}$ (Fig. 1B). Barium esophagography revealed the presence of a contrast agent in the esophagus after 5 minutes and the typical bird beak appearance (Fig. 1C). POEM treatment was decided in this patient. Under general anesthesia, intravenous antibiotics were administered prior to the procedure. A 9.8-mm-diameter high-definition gastroscope (GIF-H260; Olympus, Tokyo, Japan) was used for the POEM. A mucosal incision was made $33 \mathrm{~cm}$ from the upper incisors. Submucosal tunneling was then performed by using a dual knife at a distance of 35 to $45 \mathrm{~cm}$ from the upper incisor. We could briefly assume that the gastroscope reached the EG junction area by using several indicators such as initial narrowing point of the submucosal space of the EG junction
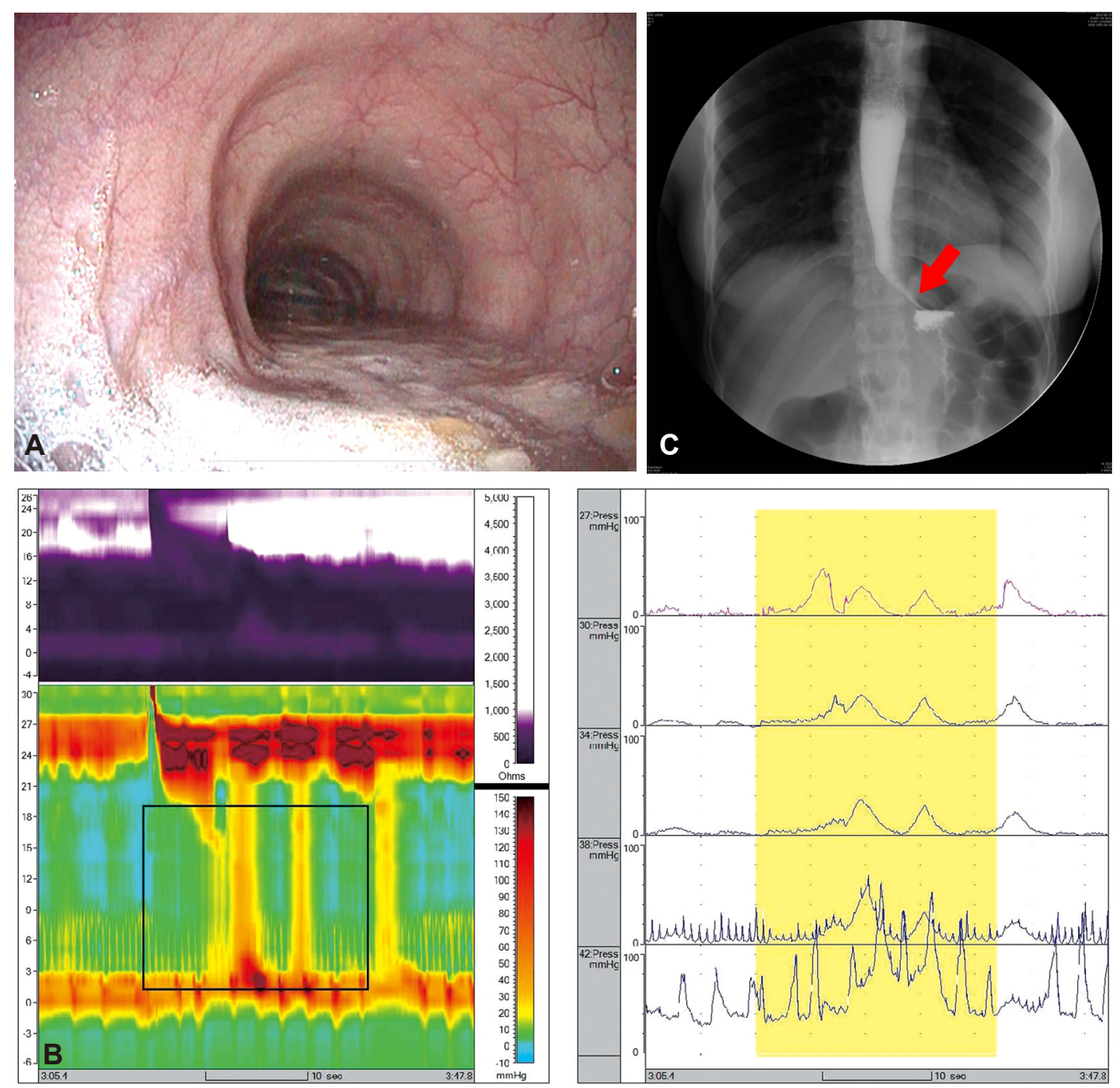

Fig. 1. (A) Initial esophagogastroduodenoscopic image showing the dilated esophagus filled with liquid and some food materials. (B) Esophageal manometric image showing elevated lower esophageal sphincter (LES) pressure and incomplete relaxation of the LES. (C) Barium esophagogram showing the typical bird beak appearance of the LES (arrow). 

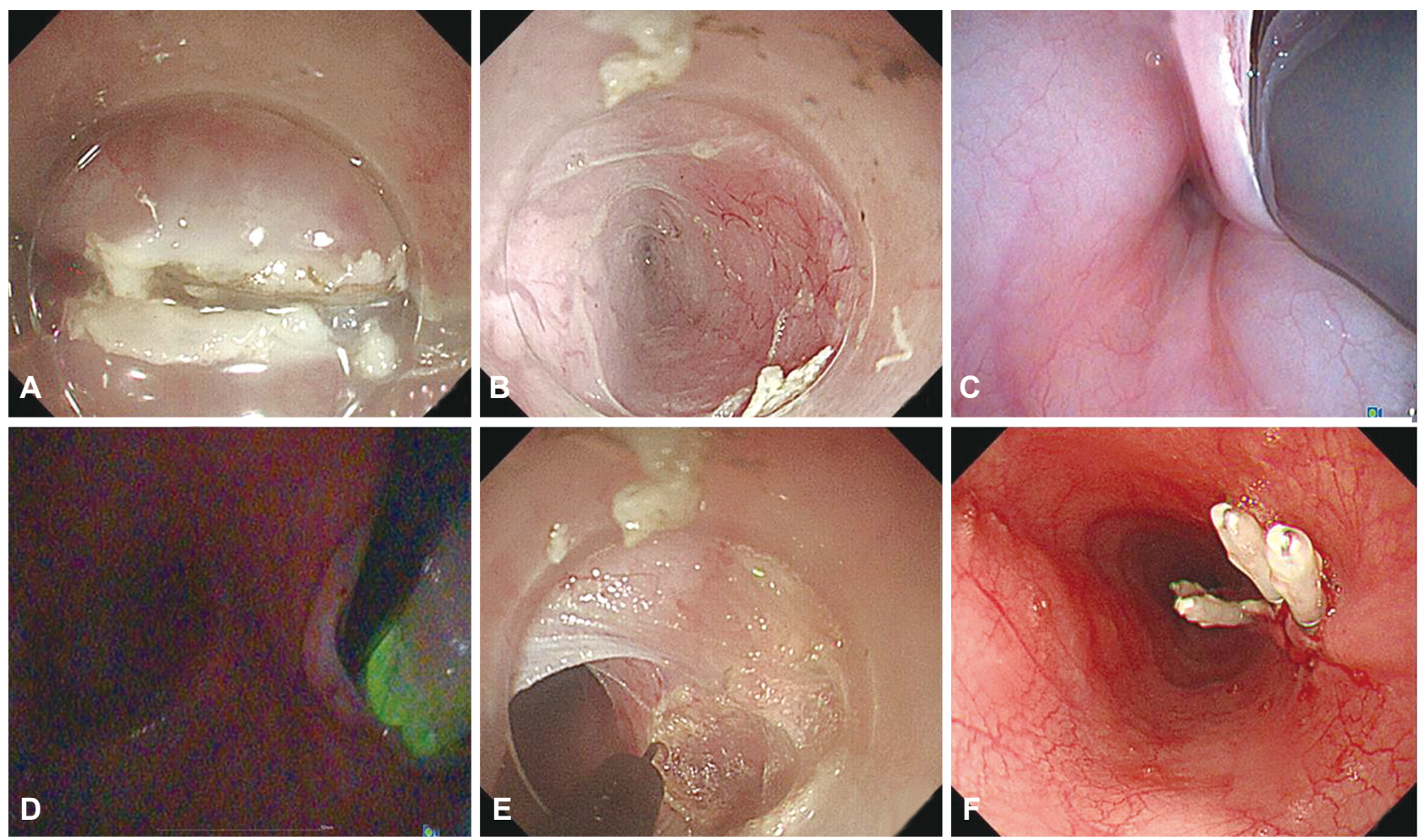

Fig. 2. The peroral endoscopic myotomy procedure. (A) A mucosal incision was performed on the mucosal surface. (B) An endoscopic tunnel is created by using a dual knife. (C) The second endoscope is introduced after formation of the submucosal tunnel. (D) We identified the extent of the submucosal tunnel by using a second endoscope and performed transillumination. (E) Endoscopic myotomy is begun by using IT and F knives. (F) After myotomy, the mucosal incision is closed by using endoscopic clips.

or visualization of a blue discoloration of the gastric cardia mucosa. When the submucosal tunnel reached around the EG junction ( $42 \mathrm{~cm}$ from the upper incisors), a $5.9-\mathrm{mm}$-diameter thin gastroscope (13820-PKS/NKS; Karl Storz, Tuttlingen, Germany) was inserted through the nose, and the position of the first endoscope was verified with transillumination to determine the exact extent of the submucosal tunneling. From the EG junction region, the tunneling proceeded while the position of the endoscope inside the submucosal tunnel was determined by using the second endoscope. The tunneling was continued under the guidance of the second endoscope and extended 2 to $3 \mathrm{~cm}$ into the cardia, after which the second thin endoscope was removed. Myotomy of the inner circular muscle was performed by using an IT knife and an F knife inside the tunnel. Finally, endo-clipping was used for closure. The entire procedure required 70 minutes to complete (Fig. 2). The patient did not develop any acute postoperative complications such as perforation or bleeding, and was subsequently discharged after the symptoms improved. Conclusively, we performed double-scope POEM for the first time and believe that it can be the ultimate procedure to cover the limitation of the current POEM.

\section{DISCUSSION}

With the recent increases in the range of endoscopic treatments available, several advances have been made in the endoscopic treatment of lesions of the submucosa or muscle. These procedures can be referred to as endoscopic submucosal surgery. POEM is considered a primary example of the treatment methods using endoscopic submucosal surgery.

However, different from that in Heller's open-eye myotomy, an important determining factor of the success in myotomy through POEM is the need to determine whether the submucosal tunnel has been appropriately extended into the gastric cardia. ${ }^{5}$ However, the current POEM procedure limits the determination of the exact extent of the submucosal tunnel.

Various ancillary methods have been developed to identify the length of submucosal tunneling. One method is the preinjection of a stain such as indocyanine green in the submucosa of the cardia. ${ }^{5}$ This method is relatively simple, but its inaccuracy is unclear. Another method is the use of the EndoFLIP system (Crospon, Carlsbad, CA, USA). This system uses impedance planimetry to measure the pre- and post-myotomy cross-sectional area and distensibility of the EG junction during the procedure. ${ }^{7}$ Although this technique is promising, only a few studies have assessed its effectiveness, necessitat- 
ing additional studies to verify its clinical usability. As the third method, endoscopic optical coherence tomography is applied. This method provides images with a depth of up to $3 \mathrm{~mm}$, a resolution of $7 \mu \mathrm{m}$, length of $6 \mathrm{~cm}$, and duration of 90 seconds, thus enabling the assessment of the condition of the esophageal muscle after POEM. ${ }^{8}$ However, this method requires additional equipment, which makes the procedure more complicated. The fourth method involves the use of fluoroscopy. In this method, fluoroscopic images are obtained by using a C-arm, by placing an endoclip on the wall opposite the EG junction, after the submucosal tunnel has been created, facilitating the determination of the distance between the endoscope tip and the endo-clip, based on which the extent of the submucosal tunnel can be calculated. ${ }^{9}$ However, the limitations of this technique include the need for a C-arm and increased exposure to radiation.

Compared with all of these trials, double-scope POEM was first applied in 2014. ${ }^{3}$ The advantages of double-scope POEM are as follows: simpler than the aforementioned methods, does not require additional equipment other than an endoscope, and easy to perform by any physician with basic endoscopy skills. In particular, the feature of double-scope POEM that allows for actual viewing is key to successful myotomy in relatively difficult cases such as type III or sigmoid type achalasia, or hiatal hernia. According to a recently published randomized controlled trial, double-scope POEM showed higher technical and clinical success rates and a lower rate of serious adverse events than single-scope POEM. ${ }^{6}$ However, in spite of these advantages, limitations remain, including longer procedure time than that of the single-scope technique, and thus further innovation is needed. The reason for the longer procedure time in the previous study, roughly 15 to 20 minutes longer procedure time, may be related to the adjunct use of the second scope for determining the length of the myotomy after completion of the myotomy. In addition, double-scope POEM provides sequential observation for POEM and is not used during POEM

Therefore, we developed the double-scope POEM technique to aid in conventional POEM, using two endoscopes simultaneously during the POEM procedure. Especially during submucosal tunneling, an orally introduced endoscope and a thin nasal endoscope are simultaneously used. As seen in the present case, the second scope was introduced immediately after the first endoscope reached around the EG junction. By inserting the second endoscope simultaneously, the submucosal tunneling can proceed in the accurate direction and with the appropriate extent. Furthermore, the entire procedure time could be reduced owing to the use of the second endoscope. In fact, in the present report, the procedure time was approximately 70 minutes, which is shorter than the average time required in the single-scope method, as reported in a previous randomized controlled trial. ${ }^{6}$

In conclusion, our double-scope POEM technique seems relatively safe and easy to perform, and can also yield better treatment outcomes, including reduced procedure time, increased efficiency, and accurate determination. Further clinical trial of double-scope POEM is needed.

\section{Conflicts of Interest}

The authors have no financial conflicts of interest.

\section{REFERENCES}

1. Vaezi MF, Richter JE. Diagnosis and management of achalasia. American College of Gastroenterology Practice Parameter Committee. Am J Gastroenterol 1999;94:3406-3412.

2. Inoue $\mathrm{H}$, Minami $\mathrm{H}$, Kobayashi $\mathrm{Y}$, et al. Peroral endoscopic myotomy (POEM) for esophageal achalasia. Endoscopy 2010;42:265-271.

3. Lee BH, Shim KY, Hong SJ, et al. Peroral endoscopic myotomy for treatment of achalasia: initial results of a korean study. Clin Endosc 2013;46:161-167.

4. Baldaque-Silva F, Marques M, Vilas-Boas F, Maia JD, Sá F, Macedo G. New transillumination auxiliary technique for peroral endoscopic myotomy. Gastrointest Endosc 2014;79:544-545.

5. Stavropoulos SN, Modayil RJ, Friedel D, Savides T. The International Per Oral Endoscopic Myotomy Survey (IPOEMS): a snapshot of the global POEM experience. Surg Endosc 2013;27:3322-3338.

6. Grimes KL, Inoue H, Onimaru M, et al. Double-scope per oral endoscopic myotomy (POEM): a prospective randomized controlled trial. Surg Endosc 2016;30:1344-1351.

7. Rieder E, Swanström LL, Perretta S, Lenglinger J, Riegler M, Dunst CM. Intraoperative assessment of esophagogastric junction distensibility during per oral endoscopic myotomy (POEM) for esophageal motility disorders. Surg Endosc 2013;27:400-405.

8. Parra V, Kedia P, Minami H, Sharaiha RZ, Kahaleh M. Endoscopic optical coherence tomography as a tool to evaluate successful myotomy after a peroral endoscopic myotomy. Gastrointest Endosc 2015;81:1251.

9. Kumbhari V, Saxena P, Messallam AA, et al. Fluoroscopy to document the extent of cardiomyotomy during peroral endoscopic myotomy. Endoscopy 2014;46 Suppl 1 UCTN:E369-E370. 